\title{
Research On Windage Yaw Of V-Type Composite Insulators In Ultra-High Voltage
}

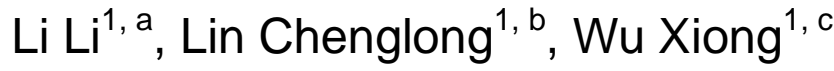 \\ ${ }^{1}$ School of Civil Engineering \& Mechanics, Huazhong University of Science and Technology, \\ Wuhan, 430074, China \\ aili2413@163.com, ${ }^{b} 793342335 @ q q . c o m,{ }^{c} 2322459481 @ q q . c o m$
}

Keywords: V-type, Composite insulator, Windage yaw, Post buckling.

\begin{abstract}
To ensure safe operation and reduce construction costs, it is necessary to study the mechanical characteristics of V-type composite insulators under windage loads, with theirs wide applications in ultra-high voltage (UHV). User-defined material (UMAT) subroutines are programmed to consider composite material parameters. Buckling characteristics of composite insulators and deformation characteristics of V-type composite insulators are analyzed based on finite element method. On this basis, V string included angle calculation method is derived considering leeward string compression, and comparison with code is conducted.
\end{abstract}

\section{Introduction}

The UHV transmission lines have the advantages of large capacity, high efficiency and long distance. They can optimize the allocation of power resources, which is of great significance for improving the economic benefit and promoting the development of clean energy. The conductors of the transmission line are usually suspended on the insulators related to the transmission towers. The insulators will swing when the transverse wind load blows on the conductors. If the swing amplitude is too large, it will cause the wind deviation to trip. Compared with the I-type, V-type insulator strings can limit the swing effectively, and reduce the size of the tower head and construction costs. At the same time, the composite insulators show a stronger capacity of compression deformation compared with porcelain and glass insulators. Therefore, the application of V-type composite insulators in engineering becomes more and more extensive [1-5]. To ensure security of operation and reduce construction costs, it is necessary to study the mechanical characteristics of V-type composite insulators under wind loads in UHV.

The mechanics characteristics and design method of V-type composite insulator strings under wind load have been studied by domestic and foreign scholars. Shen Dingshen et al. [6] studied the effect of long-term compression of composite insulator on the mechanics performance of the insulator, and experiment about the mechanics performance of V-type insulators. By analyzing the dynamic load characteristics of V-type $330 \mathrm{kV}$ composite insulator strings, Hou Lei et al. [7] find that the dynamic analysis should be done when the compression performance of the insulator string on the leeward side was taken into consideration for insulators design. Boddy et al. [8] summarized the design methods of foreign V-type insulators, and studied the influences of wire types, wind load, vertical span and V string angle on windage. It is given that the influence of various factors should be considered in the design of V-type insulator. Liao Yongli et al. [9, 10] established V-type composite insulator strings model of the $750 \mathrm{kV}$ transmission line by using the finite element method, analyzed the deformation characteristics of the V-type strings in different included angle and compared it with the test results.

Among the above researches, the mechanical and deformation characteristics of the V-type composite insulator strings have been studied. However, a few people have studied the post buckling behavior of composite insulator strings and its influence on the design of V-type composite insulator strings.

This paper thus focuses on the post buckling characteristics of composite insulators and its effect 
on the design of V strings' included angle. Firstly, the UMAT subroutine of composite material was programmed to consider the anisotropy of material. Then the post buckling behavior of composite insulator was analyzed by the finite element method. On the basis of this, the research on the windage yaw characteristics of the $\mathrm{V}$ string was carried out. Finally the calculation method the V string included angle is derived considering leeward string's compression.

\section{The Analysis of Composite Insulators}

Subroutine for Composite Mandrel Material. The composite insulator is mainly composed of a mandrel and shed which mainly bear the electrical load and can be neglected in the force analysis. The mandrel is made of glass fiber and epoxy resin and the material properties are typical composite materials. To consider the influence of the mandrel material parameters on the force, UMAT subroutine was written in abaqus. So that the orthorhombic anisotropy and the different properties of tension and compression can be considered.

In abaqus The UMAT's procedure is that when the result of step $\mathrm{n}$ of $\sigma_{n}$ and $\varepsilon_{n}$, and the next strain increment $d \varepsilon_{n+1}$ is given, The new stress $\sigma_{n+1}$ can be calculated by calling the Jacobian matrix $\operatorname{DDSDDE}(I, J)=\partial \Delta \sigma / \partial \Delta \varepsilon$ of the material in UMAT. Therefore, it is necessary to determine the constitutive equation of the material, i.e. the Jacobian matrix.

The mandrel is simplified to orthotropic materials, which has different properties of tension and compression different material parameters, the constitutive relationship is showed as follows:

$$
\left\{\begin{array}{l}
\varepsilon_{11} \\
\varepsilon_{22} \\
\varepsilon_{33} \\
\gamma_{12} \\
\gamma_{13} \\
\gamma_{23}
\end{array}\right\}=\left[\begin{array}{cccccc}
1 / E_{1} & -v_{21} / E_{2} & -v_{31} / E_{3} & 0 & 0 & 0 \\
-v_{12} / E_{1} & 1 / E_{2} & -v_{32} / E_{3} & 0 & 0 & 0 \\
-v_{13} / E_{1} & -v_{23} / E_{2} & 1 / E_{3} & 0 & 0 & 0 \\
0 & 0 & 0 & 1 / G_{12} & 0 & 0 \\
0 & 0 & 0 & 0 & 1 / G_{13} & 0 \\
0 & 0 & 0 & 0 & 0 & 1 / G_{23}
\end{array}\right]\left\{\begin{array}{l}
\sigma_{11} \\
\sigma_{22} \\
\sigma_{33} \\
\sigma_{12} \\
\sigma_{13} \\
\sigma_{23}
\end{array}\right\}
$$

In practical application, due to too many parameters in formula (1), according to the nature of the mandrel, the formula can be simplified as follows: with the glass fiber as the spindle direction 1, $\mathrm{E}$ is decided by glass fiber modulus; with the epoxy resin as spindle direction 2, 3, so $\mathrm{E}_{2}$ is equal to $\mathrm{E}_{3}$; Poisson ratio is $v_{12}=v_{21}=v_{13}=v_{31}=v_{23}=v_{32}=v$. In this study, the mandrel is not subjected to the tangential force, $G_{12}=G_{13}=G_{23}=G$. The resulting Jacobian matrix can be simplified as follows:

$$
J=\left[\begin{array}{cccccc}
(v-1) E_{1} / \lambda & -v E_{1} / \lambda & -v E_{1} / \lambda & 0 & 0 & 0 \\
-v E_{2} / \lambda & (v-1) E_{2} / \lambda & -v E_{2} / \lambda & 0 & 0 & 0 \\
-v E_{2} / \lambda & -v E_{2} / \lambda & (v-1) E_{2} / \lambda & 0 & 0 & 0 \\
0 & 0 & 0 & G & 0 & 0 \\
0 & 0 & 0 & 0 & G & 0 \\
0 & 0 & 0 & 0 & 0 & G
\end{array}\right]
$$

Where $\lambda=2 v^{2}+v-1$, tension or compression Jacobian matrix can be obtained by using different $E_{1}$ values. The process to achieve the tension and compression properties of the composite material is showed in the Fig. 1. This UMAT subroutine will be used for the following finite element analysis. 


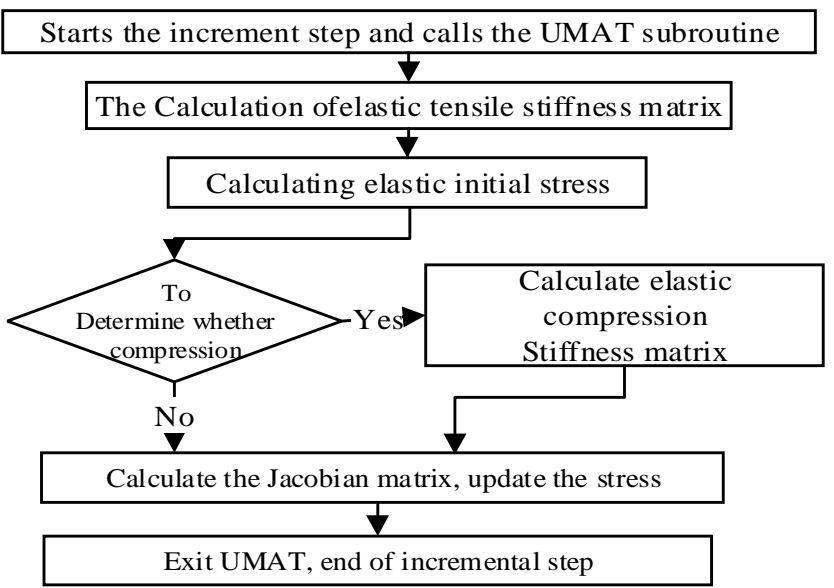

Fig. 1 Flow chart of UMAT.

The Post Buckling Analysis of Mandrel. The composite insulation type studied in this paper is FXBW-1000/300, and the shed isn't considered in the analysis as mentioned above. According to paper $[11,12]$, the parameters of the mandrel are shown in Table 1.

Table 1. Parameters of composite insulators.

\begin{tabular}{c|c}
\hline Parameter name & Parameter values \\
\hline Axial tensile elastic modulus & $40 \mathrm{GPa}$ \\
\hline Axial compressive elastic modulus & $40 \mathrm{GPa}$ \\
\hline Transverse elastic modulus & $8.4 \mathrm{GPa}$ \\
\hline Shear modulus & $4 \mathrm{GPa}$ \\
\hline Poisson ratio & 0.3 \\
\hline Length of mandrel & $9.0 \mathrm{~m}$ \\
\hline Mandrel diameter & $40 \mathrm{~mm}$ \\
\hline The quality of the mandrel & $21.5 \mathrm{~kg}$ \\
\hline
\end{tabular}

According to the stability theory, when one end is hinged and one end is sliding support (Fig. 2), the eigenvalue buckling load of the common beam element mandrel is calculated as shown in equation (3):

$$
P_{c r}=\frac{\pi^{2} E I}{(\mu l)^{2}}=\frac{\pi^{2} \times 40 e 9 \times \pi \times 0.04^{4}}{(1 \times 9)^{2} \times 64}=612.47 \mathrm{~N}
$$

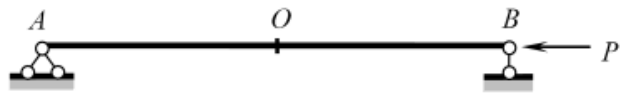

Fig. 2 Bearing schematic of composite insulator.

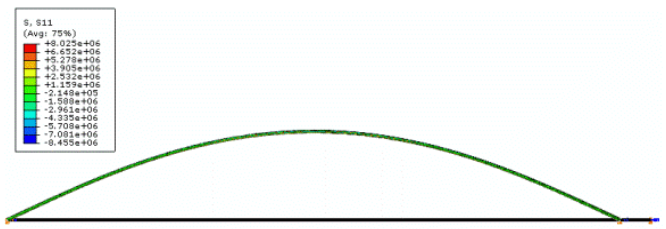

Fig. 3 Deformation of composite insulator.

To finish the establishment of three-dimensional finite element model of mandrel in Abaqus, firstly, the modal analysis is carried out. A buckling load of $597.78 \mathrm{~N}$ is gotten with some errors with the theoretical results, for the finite element method considering the influence of parameters of composite materials. The nonlinear buckling analysis was carried out using the Riks method with the initial defect L/1000, and the deformation of the mandrel is shown in Fig. 3. The lateral displacement and the external load of the Intermediate point $O$ point are shown in Fig. 4 and the maximum stress of the $\mathrm{O}$ point section and the horizontal displacement of the $\mathrm{B}$ point are shown in Fig. 5. 


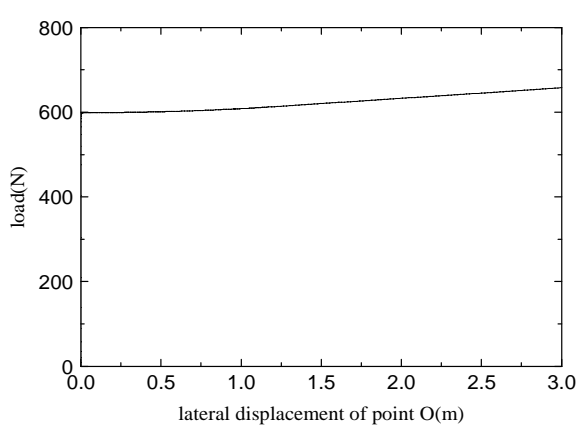

Fig. 4 Relationship of lateral displacement of $\mathrm{O}$ with load.

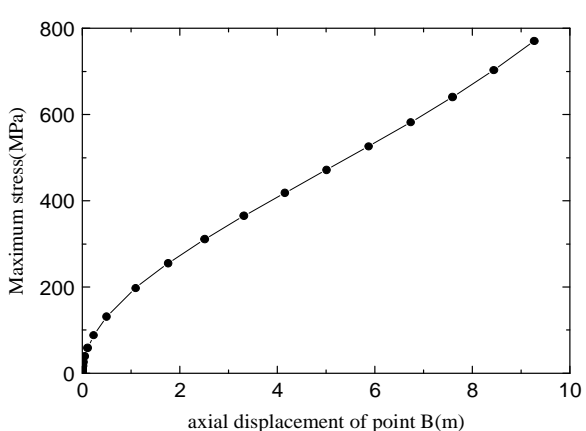

Fig. 5 Relationship of maximum stress of $\mathrm{O}$ with axial displacement of $\mathrm{B}$.

Previous studies have shown that the composite insulator mandrel failure stress is between $800 \mathrm{MPa} 1000 \mathrm{MPa}$. This paper takes $800 \mathrm{MPa}$ as the maximum failure stress of mandrel. According to the paper [13], the safety coefficient of insulator mechanical strength is 4 . Therefore $800 / 4=$ $200 \mathrm{MPa}$ is taken as the maximum compression stress. From Fig. 5, the horizontal displacement of the point $\mathrm{B}$ is $1.26 \mathrm{~m}$ when the mandrel reaches the control stress.

\section{Calculation of Windage Yaw of V-type Composite Insulator Strings}

Introduction to Theoretical Methods. At present, the design procedure of transmission line [14] in our country is to prevent the compression of insulator when calculating the stress of V strings, with the mechanical simplified model shown in Fig. 1. The insulator strings is simplified as a rigid body with no deformation, and the main load is the wind load $\mathrm{W}_{\mathrm{H}}$ and the gravity load of the conductor $\mathrm{G}_{\mathrm{V}}$. Because the weight of the composite insulator is small, the gravity effect can be neglected when the windage yaw is calculated. Under the effect of the maximum windage load and the gravity load of the conductor, the minimum design concluded angle $\theta$ of the $\mathrm{V}$ string can be obtained by the static equilibrium, such as the formula (4).

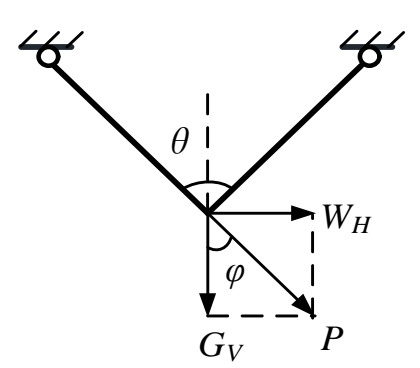

Fig. 6 Mechanical model of V-type windage.

$$
\begin{aligned}
& \theta=2 \arctan \left(W_{H} / G_{V}\right)=2 \varphi \\
& W_{H}=\frac{1}{1.6} \alpha n \mu_{s c} \mu_{z} d l_{H} V^{2} ; G_{V}=n q l_{V}
\end{aligned}
$$

Where $\alpha$ is wind pressure inhomogeneity coefficient, $\mathrm{n}$ is wire splitting number, $\mu_{s c}$ is wire shape coefficient, $\mu_{z}$ is wind pressure height coefficient, $d$ is wire diameter, $l_{H}$ is horizontal span, $V$ is design wind speed, $q$ is wire weight, $l_{V}$ is the vertical span. The above mentioned method didn't consider the influence of insulator.

Paper [15] provides that the half of the concluded angel of v-type composite insulators can be 
$5^{\circ}-10^{\circ}$ less than the maximum windage angle, but didn't give a specific method. This paper is based on the windage research of FXBW-1000/300 v-type composite insulators, the string is $8 \times \mathrm{JL} / \mathrm{G} 1 \mathrm{~A}-630 / 45$, the span is $600 \mathrm{~m}$, and the ground roughness is Class B.

Analysis of V-type Composite Insulator Strings windage. This paper is based on the FXBW-1000/300 v-type composite insulators, $8 \times$ JL / G1A-630/45 wire, 600m span, Class B of the ground roughness.

The finite element model of three dimensional V-type composite insulator was established by taking the angle of the $\mathrm{V}$ string as $70^{\circ}, 80^{\circ}, 90^{\circ}, 100^{\circ}$, to study the force and deformation characteristics under the self-weight and wind load respectively. By formula (2) and (3) the wire load can calculate as $G_{V}=97.81 \mathrm{kN}$. When wind speed is $30 \mathrm{~m} / \mathrm{s}$, and height variation factor of wind pressure is 1 , the wind load is $W_{H}=61.24 \mathrm{kN}$. The variation of the horizontal displacement and the vertical displacement of the $\mathrm{V}$-type insulator string at different angles with the wind deflection load is shown in Fig. 7 and Fig. 8.

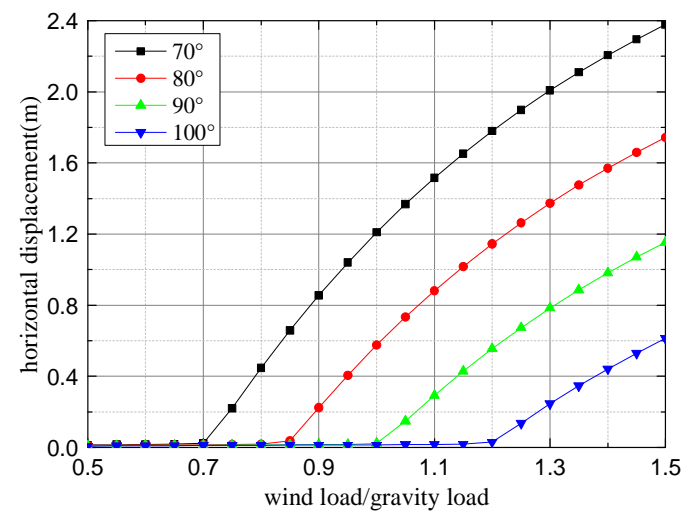

Fig. 7 Bottom horizontal displacement under different angles.

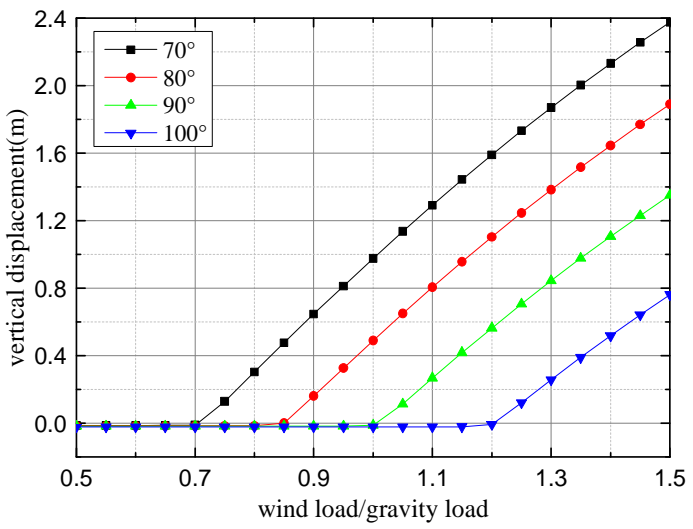

Fig. 8 Bottom vertical displacement under different angles.

It can be seen from the figure that the horizontal and vertical displacements of the insulator at the bottom of V-string have a critical wind load value. When the wind load is less than the critical value, the horizontal and vertical displacement of the insulator are small. At this time the leeward side of the insulator is in the unload stage, the axial tension is getting smaller and smaller. When the wind load is greater than the critical value, the insulator displacement increases rapidly with the increase of the wind load, meanwhile the load on the leeward side of insulator unload completely, the external force is mainly borne by the windward side insulator. The relationship between the critical windage angle and the angle $\theta$ is shown in Table 2 . The results show that the critical windage angle of $\mathrm{V}$ string is approximately equal to half of the angle of V-type composite insulator string.

Table 2. Relationship of critical windage angle with angles.

\begin{tabular}{cccc}
\hline V string angle & Critical windage angle & Half the angle & relative error \\
$\theta$ & $\varphi_{\max }$ & $0.5 \theta$ & $\%$ \\
\hline 70 & 35.1 & 35.0 & 0.29 \\
80 & 40.1 & 40.0 & 0.25 \\
90 & 45.1 & 45.0 & 0.22 \\
100 & 50.1 & 50.0 & 0.20 \\
\hline
\end{tabular}

When the included angle is $90^{\circ}$, the deformation of the insulator string under the action of $40 \mathrm{~m} / \mathrm{s}$ wind speed is shown in Fig. 9. It can be seen from the figure, that the leeward side of insulator was buckling and the maximum stress section is $89.1 \mathrm{MPa}$. It can be seen from Section 2.2 that the buckling load of the insulator is less than $1 \mathrm{kN}$, which is much less than the external force. 


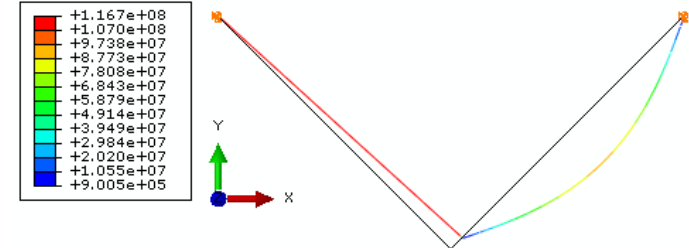

Fig. 9 Deformation of $90^{\circ} \mathrm{V}$ string under velocity of $40 \mathrm{~m} / \mathrm{s}$.

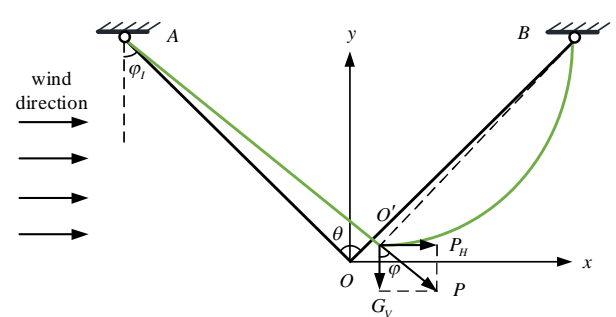

Fig. 10 Windage model of $\mathrm{V}$ string.

From the analysis of composite insulator string and V-type composite insulator string buckling analysis of the deformation characteristics, it can be seen that the effect of the leeward side of the insulator on the $\mathrm{V}$ string windage yaw can be ignored, at this time, the windward side insulator windage angle can be calculated by formula (6).

$$
\varphi_{I}=\varphi=\arctan \left(W_{H} / G_{V}\right)
$$

When the maximum windage yaw is controlled by the compressive strength of the insulator on the leeward side, the maximum buckling deformation at the leeward side is $\Delta l=1.26 \mathrm{~m}$, according to the analysis in Section 2.2. As shown in Fig. 10, supposing the coordinate of the bottom $O^{\prime}$ is (x, $\mathrm{y}$ ), and the $\mathrm{x}$ and $\mathrm{y}$ can be obtained from $O^{\prime} A=l$ and $O^{\prime} B=l-\Delta l$, such as equation (7). From equation (6), we can get the relationship between the angle of $\mathrm{V}$ string and windage load when the wind string is under pressure, such as equation (8). By equation (8) minimum design angle of V-type composite insulator string $\theta_{\min }$ can be deduced by using Newton method to design wind load $W_{H}$ and $G_{V}$, considering the leeward side insulator pressure.

$$
\begin{aligned}
& x=\frac{2 l \Delta l-\Delta l^{2}}{4 l \sin (\theta / 2)} \\
& y=\arctan \frac{x+l \sin (\theta / 2)}{l \cos (\theta / 2)-y}+\sqrt{l^{2}-[x+l \sin (\theta / 2)]^{2}} \\
& \varphi_{\max }=\arctan \frac{W_{H}}{G_{V}}=\arctan \frac{x+l \sin (\theta / 2)}{l \cos (\theta / 2)-y}
\end{aligned}
$$

Under certain windage load, the minimum $\mathrm{V}$ string angle $\theta$ which the insulator of the leeward side isn't under pressure, the minimum angle $\theta_{\min }$ of the $\mathrm{V}$ string which the insulator of the leeward side can be under pressure and the corresponding reduced angle are shown in Table 3. It can be seen that when considering the compression of the composite insulator string on the leeward side, half of

\begin{tabular}{|c|c|c|c|}
\hline $\begin{array}{c}\text { Design wind load } \\
W_{H} / \mathrm{kN}\end{array}$ & $\begin{array}{c}\text { Angle of norm } \\
\theta\end{array}$ & $\begin{array}{c}\text { Minimum angle } \\
\theta_{\min }\end{array}$ & $\begin{array}{c}\text { Difference } \\
0.5 \theta-0.5 \theta_{\text {min }}\end{array}$ \\
\hline 68.59 & 70 & 49.6 & 10.3 \\
\hline 82.14 & 80 & 62.3 & 8.9 \\
\hline 97.82 & 90 & 73.5 & 8.3 \\
\hline 116.70 & 100 & 84.0 & 8.0 \\
\hline
\end{tabular}
the included angle of the $\mathrm{V}$ string can be about $8^{\circ}$ less than the maximum windage angle, which is consistent with the $5^{\circ} \sim 10^{\circ}$ specified by the circuit design specification. Equation (9) provides a specific calculation method to consider the leeward string is under pressure.

\section{Conclusion}

In this paper, the post buckling characteristics of composite insulators were analyzed. The 
mechanical and deformation characteristics of windage yaw are studied by the finite element method. Moreover, the calculation method for the concluded angle of $\mathrm{V}$ string is derived considering leeward string compression. And the result was compared with the result from code. The main are summarised as follows:

(1)Composite insulators have a large deformation capacity and a small buckling load.

(2)While considering leeward string compression, the half of the V-type insulator string's included angle can be 8 degrees less than maximum windage angle.

\section{Reference}

[1] Z. C. Guan, G. M. Peng, L. M. Wang, et al. Application and Key Technical Study of Composite Insulators, High Voltage Eng. 37(3) (2011) 513-519.

[2] Z. Y. Su, Y. Yin, J. Zhou, et al. Reliability of Composite Insulators Used for UHV AC/DC Transmission Lines, High Voltage Eng. 35(10) (2009) 2329-2334.

[3] Z. H. Liu, Present Situation and Prospects of Applying Composite Insulators to UHF Transmission Lines in China, Power Syst. Technol. 30(12) (2006) 1-7.

[4] J. Yuan, Long-Term Mechanical Property of Composite Insulators for UHV Transmission Lines, Power Syst. Technol. 30(12) (2006) 29-32.

[5] Z. Lin, D. M. Xu, Technical and Economical Comparison of V - shape Insulator Strings Used for Middle Phase of 500 kV Suspension Towers, Electric Power Construct. 18(3) (1997) 27-29.

[6] D. S. Shen, W. Cai, J. Wang, Investigation on Application of Composite Insulator for "V" String, Insulators Surge Arresters, (5) (2004) 14-16.

[7] H. Lei, W. Liming, G. Zhicheng, Dynamics characteristic of V-string composite insulators for 330kV overhead transmission line, Proc IEEE Conf., 2006, 429-432.

[8] D. M. Boddy, V-String Swing Angle Derivation, Design Considerations, and Structure Design Impacts, Electric. Transm. Substation Struct. (2012) 119-131.

[9] Y. Liao, L. Hou, L. Wang, et al. Included Angle Selection of V-Shape Insulator String for 750-kV Compact Transmission Line, IEEE Trans. Power Deliv. 26(1) (2011) 385-393.

[10] Y. L. Liao, L. M. Wang, Y. W. Zhang, Analysis of Windage Yaw Deformation of Composite Insulators in Compact Transmission Lines, South. Power Syst. Technol. 4(3) (2010) 77-81.

[11] L. F. Cai, Y. P. Liu, Y. Chen, et al. Research on Buckling Characteristics of V-shaped Composite Insulators Mandrel Based on ANSYS, North China Electric Power, (6) (2014) 6-9.

[12] M. Kumosa, Y. Han, L. Kumosa, Analyses of composite insulators with crimped end-fittings: Part I—non linear finite element computations, Compos. Sci. Technol. 62(9) (2002) 1191-1207.

[13] GB 50545. Code for Design of 110kV 750kV Overhead Transmission Line, Beijing: China Planning Press, 2010.

[14] D. S. Zhang, Design Manual for High Voltage Transmission Line, Beijing: China Electric Power Press, 2003. 